\title{
The continuing debate over the structure of cellulose: historical perspective and outlook
}

\author{
Henri Chanzy
}

Received: 4 April 2011/Accepted: 6 April 2011/Published online: 21 April 2011

(C) Springer Science+Business Media B.V. 2011

The crystalline structure of native cellulose, with its unique microfibrillar morphology resulting from a complex biogenesis mechanism, has not only been the most studied subject in polymer science, but is still generating great interest along with lively debates and controversies. This issue of Cellulose features such a debate in the form of two articles dealing with the conformational analysis of the glucosidic bond in the cellulose chains. This debate is but the latest incident in a long string of events, which represent the foundation of our current understanding of this important material. This makes this an opportune moment to reflect on some of the major milestones in the history of cellulose structure evaluation, which also often involved debates and controversies.

It was in 1913, i.e. close to one century ago, that Nishikawa and Ono showed the first diffraction patterns of two cellulose-based plant materials, namely bamboo and hemp, taking advantage of the discovery of the interference phenomena of X-rays reported 1 year earlier by Laue, Friedrich and

H. Chanzy-affiliated with the Universite Joseph Fourier and member of the Institut de Chimie Moléculaire de Grenoble.

H. Chanzy $(\bowtie)$

Centre de Recherches sur les Macromolécules Végétales (CERMAV-CNRS), BP 53, 38041 Grenoble cedex 9,

France

e-mail: Henri.Chanzy@cermav.cnrs.fr
Knipping. Following this early report, diffraction diagrams of a large number of cellulose materials were duly recorded and subjected to analysis, as it was felt that the knowledge of the crystalline structure of cellulose was of great importance for the development and improvement of cellulose-based products. While recording diffraction patterns of these specimens, it was soon realized that even the X-ray data sets of the most crystalline samples remained limited and it was therefore hard to deduce from them alone the required structural details. It is only when the diffraction data could be complemented by the occurrence of new techniques that substantial advances toward the structural description of cellulose could be made. The sequential emergence of such techniques explains the succession of steps leading to the present understanding.

In the progress toward the molecular description of the crystal structure of cellulose from X-ray diffraction data, a first landmark was the incorporation of the stereochemistry of the glucosyl moieties in the molecular models built by, among others, Meyer and Mark (1928), later revised by Meyer and Misch (1937), who presented a model where the crystal of cellulose consisted of two antiparallel chains. This last model, which remained the established standard for at least two decades, was confronted with a series of conflicting data, resulting from new techniques. In 1958, the recording of electron diffraction diagrams of frozen Valonia cell wall fragments allowed Honjo and Watanabe to obtain diffraction data sets far better 
resolved than any X-ray diagram recorded earlier. When indexed, these diagrams required unit cell parameters twice as large as those of the Meyer and Misch structure, thus leading to an eight-chain unit cell. In 1974, the input of conformational analysis supported by the dissemination of computers, led the groups of Sarko and Blackwell to show that the crystal structure of Valonia cellulose consisted of a parallel chain arrangement, in disagreement with the Meyer and Misch antiparallel model. Still the Sarko and Blackwell models were based on a two-chain system, but the debate turned about the fine details of the "parallel-up" and "parallel-down" possibilities while there was an agreement on the probable $t g$ conformation of all hydroxymethyl groups in the cellulose crystal.

In the early 1980 , the emerging ${ }^{13} \mathrm{C}$ CP-MAS spectroscopy became a determinant technique to add new dimensions to the results from diffraction data. In 1983, the exploitation of cellulose spectra in light of those of simple sugars, led Horii et al. to confirm the $t g$ conformation of the hydroxymethyl moieties in native cellulose. A totally unexpected development occurred in 1984 when, in their seminal paper, Atalla and VanderHart clearly demonstrated that their NMR data indicated that native cellulose was in fact a composite of two distinct allomorphs, namely cellulose $\mathrm{I} \alpha$ and cellulose I $\beta$. Samples such as those of Valonia and bacterial cellulose were rich in $\mathrm{I} \alpha$, whereas cotton and ramie were dominated by the $\mathrm{I} \beta$ phase. Using the same technique, Belton et al. showed in 1989 that tunicin, another highly crystalline cellulose sample could be considered as the almost pure $\mathrm{I} \beta$ standard. For a few years, the ${ }^{13} \mathrm{C} \mathrm{CP}$ MAS data were regarded with some doubts by the crystallographer community, but in 1990 and 1991,
Sugiyama et al. reconciled everybody by recording conclusive electron micro-diffraction diagrams where separate domains, only containing the $\mathrm{I} \alpha$ or $\mathrm{I} \beta$ phase could clearly be identified (Fig. 1). The I $\alpha$ phase consisted of a one-chain triclinic P1 structure, whereas in the $\mathrm{I} \beta$ phase, there were two chains organized along a $\mathrm{P} 2{ }_{1}$ monoclinic space group. In cellulose samples such as those of Valonia and other seaweeds, the two phases could be visualized as distinct entities in any given microfibril. While tunicin had proven to be essentially of the $1 \beta$ phase, the cell wall of Glaucocystis was almost of pure $\mathrm{I} \alpha$, and therefore could be considered as the standard of the other allomorph.

Concomitant to these progresses, transmission electron microscopy (TEM) operated in normal or diffraction contrast imaging mode, together with electron diffraction, played another important role to complement the X-ray analysis of cellulose. As early as 1984 Sugiyama et al. produced images showing the lattice of cellulose in Valonia. The parallelism of the cellulose chains within Valonia microfibrils and nanocrystals was confirmed by Chanzy and Henrissat in 1985, by recording TEM images where these elements were unidirectionally degraded by a cellulase, specific for the digestion of cellulose from its non-reducing end. Another important result was published in 1997 when the group of Sugiyama could demonstrate by TEM methods, not only that the crystal of cellulose was of the parallel-up type, but also that the biogenesis of the cellulose microfibrils resulted from the insertion of the cellulose precursors at its non-reducing end.

A further advance toward the resolution of the crystal structure of cellulose has resulted from the diffraction work of Nishiyama et al. during the period
Fig. 1 Historical moment at CERMAV in 1990, when Junji Sugiyama identified the $\mathrm{I} \alpha$ and $\mathrm{I} \beta$ phases of cellulose by micro electron diffraction of a Microdictyon cell wall fragment
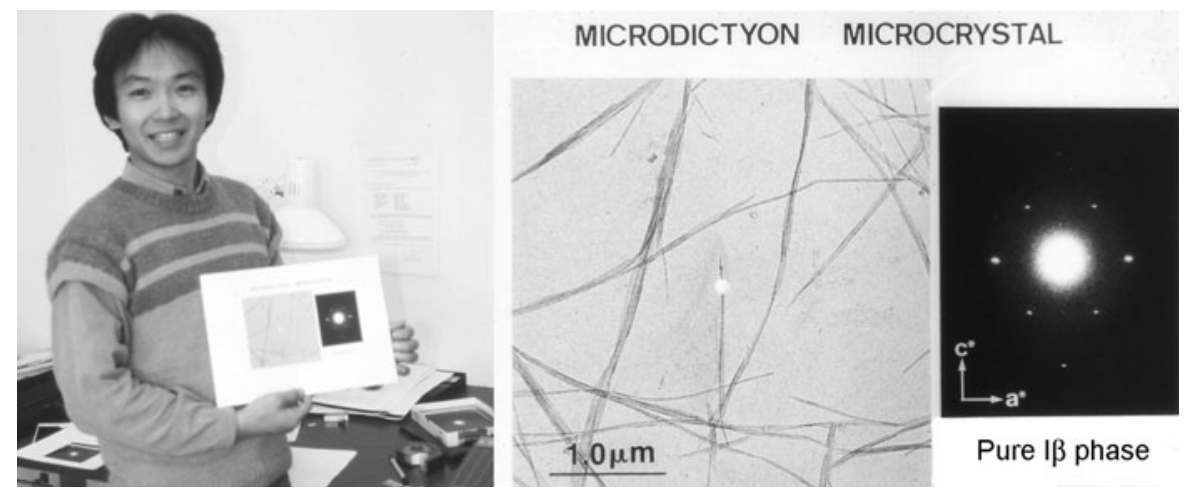

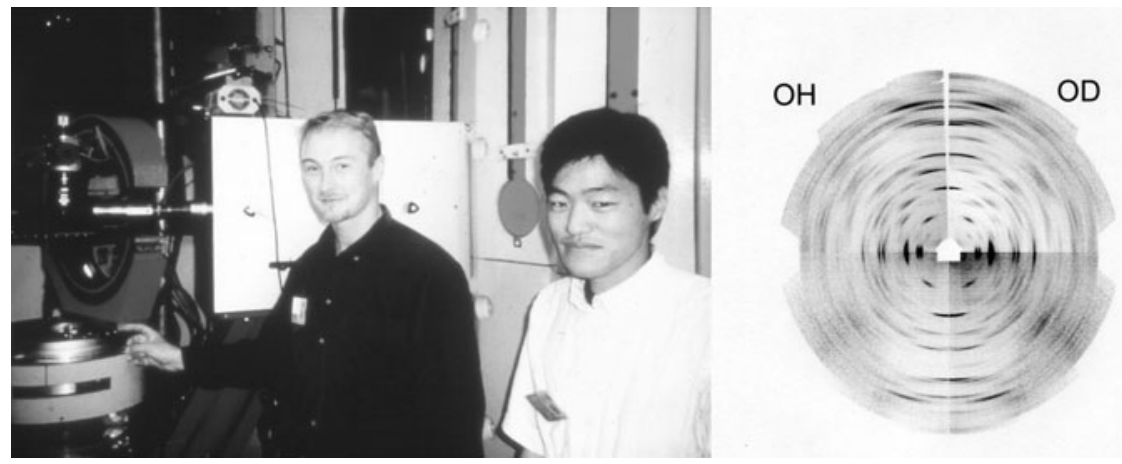

Fig. 2 The recording of hydrogenated and deuterated high-resolution neutron fiber diffraction patterns of I $\beta$ cellulose by Paul Langan and Yoshiharu Nishiyama at ILL, Grenoble in 2002

1997-2003. The success of their diffraction work resulted in part from the preparation of oriented mats of cellulose nanocrystals from either tunicin or Glaucocystis cell wall. In addition, these authors implemented a method whereby the $\mathrm{OH}$ moieties in the crystalline core of these nanocrystals could be deuterated in a stable manner, without losing the crystalline integrity of the specimens. By analyzing these samples by state-of-the-art synchrotron X-ray and using the expertise developed by Langan for neutron diffraction experiments and analysis, well resolved crystal structures of cellulose $\mathrm{I} \alpha$ and $\mathrm{I} \beta$ could be obtained (Fig. 2). With neutron data, the difference in the intensities of the neutron data between hydrogenated and deuterated samples, led to the description, at the atomic level, of the hydrogen bonds holding the crystalline structures together.

While it seems today that the main part of the secret code of the cellulose crystal has been cracked, there are still many aspects of the cellulose ultrastructure that need the implementation of new tools. Among the major questions awaiting answers, the structural details of the cellulose microfibril surface still need to be described. In fact, despite promising, but never reproduced, high-resolution atomic force microscopy (AFM) images of Valonia microfibrils published by Baker et al. in 1998 and 2000, we still do not know whether the cellulose surface is as highly organized as these images tended to show. In this line, the rather broad resonance signals attributed to the surface chains in the ${ }^{13} \mathrm{C}$ CP-MAS data has not been fully accounted for. Hydrophobic surface domains, which are critical for the adsorption of the cellulose binding modules of cellulases, have been located by TEM at the corner of the cellulose microfibrils. The sharpness of these corners, together with the percentage of hydrophobic surfaces with respect to the hydrophilic surfaces remain to be ascertained, not only in the Valonia model, but also in the less crystalline celluloses such as those of wood or cotton.

Questions of the so-called "amorphous phase" of cellulose as well as that of the crystalline defects are other important issues, where only fragmentary knowledge exists so far. In 2003, Nishiyama et al. have revealed the occurrence of a small-angle neutron scattering (SANS) meridional maximum at $150 \mathrm{~nm}$, by subtracting the SANS pattern of deuterated ramie from that of its hydrogenated counterpart. If one assumes a distance of $0.5 \mathrm{~nm}$ per glucosyl residue, the periodicity of $150 \mathrm{~nm}$ corresponds to a regular longitudinal succession of crystalline cellulose segments with a degree of polymerization (DP) of 300 , connected to one another by less organized water receptive domains. The value of 300 , which is exactly that of the DP of ramie after hydrolysis with hot $4 \mathrm{~N} \mathrm{HCl}$ for $7 \mathrm{~h}$, confirms the periodicity of acidsensitive crystalline defects along the ramie microfibrils. Systematizing such experiments with other cellulose samples and coupling the results with highresolution AFM images should lead to a better perception of the structural defects of cellulose. Their identification as twists, kinks or chain ends etc., is important since these defects are believed to be the preferential reactive sites for the initial interaction of cellulose with external reagents. In terms of mechanical properties, these defects are also worth describing, since they act as intrinsic weak points. 
One cannot talk about future improvements in the knowledge of the fine structure of cellulose without mentioning the potential of simulation, and in particular that of molecular dynamics (MD). In fact, this approach is becoming quite important and one can forecast that it may become a key structural technique, when computing time will no longer be a limiting factor, and when the various modeling strategies have reached their maturation. At present, the current MD literature is animated by debates about the selection of models based on either infinite crystalline phases or, alternately, on thin and short crystals. Whereas the former choice is adequate to describe the cellulose bulk, the latter is more appropriate to predict the observed microfibrillar helical twists. At present, it is not yet clear whether any of these alternate models or a superior new one will be able to account for the full details of the slender cellulose microfibril: its crystalline perfection and stability, its imperfections, together with the molecular description of its surface. In addition to this uncertainty, it seems that the current empirical force fields do not apply too well to the unique inner cohesion of the cellulose crystal and that an "ultimate force field", applicable to cellulose materials still needs to be determined. Full ab initio calculations that would overcome the empiricism of the force fields are not yet feasible, due to computing limitations, but should become available in the future.

In the present issue of Cellulose, it can be seen that debates about modeling methodologies are not limited to the ultrastructural aspects of cellulose, but also touch on the description of the flexibility of the glucosidic linkage of cellobiose, one of the basic conformational features of cellulose. It is this flexibility after all that plays a crucial role in directing the incipient state of intermolecular associations. 